\title{
Subchondroplasty for Treating Bone Marrow Lesions
}

\author{
Steven Brad Cohen, MD ${ }^{1}$ Peter F. Sharkey, MD ${ }^{1}$ \\ ${ }^{1}$ Department of Orthopedic Surgery, Rothman Institute, Thomas \\ Jefferson University, Philadelphia, Pennsylvania \\ J Knee Surg 2016;29:555-563.

\begin{abstract}
Address for correspondence Steven Brad Cohen, MD, Department of Orthopedic Surgery, Rothman Institute, Thomas Jefferson University, 925 Chestnut Street, Philadelphia, Pennsylvania 19107 (e-mail: steven.cohen@rothmaninstitute.com).
\end{abstract}

Abstract
Keywords
- arthritis
- arthroscopy
- bone marrow edema
- bone marrow lesion
- subchondroplasty

The prognosis of osteoarthritis $(\mathrm{OA})$ is worsened by persistent subchondral defects known as bone marrow lesions (BMLs), which herald severe joint degeneration and the need for joint replacement. Joint-preserving treatments that reverse the progression of pain and immobility are limited. Subchondroplasty is a procedure developed to treat BMLs by injecting a calcium phosphate bone substitute into compromised subchondral bone, under fluoroscopic guidance. Here we evaluate the effectiveness of this approach for relieving pain and improving function in patients with documented BMLs and advanced knee $\mathrm{OA}$, in a retrospective study. Data were collected from a consecutive patient series $(n=66)$ who underwent subchondroplasty combined with arthroscopy, performed at a single center by one surgeon. We observed significant improvements in both pain and function following subchondroplasty with arthroscopic debridement, as measured by the visual analog scale (VAS) and the International Knee Documentation Committee (IKDC) Subjective Knee Evaluation Form, through 2 years postoperative follow-up. Given that arthroscopic debridement alone has been previously shown to yield insignificant pain relief beyond 6 months postoperatively, our results suggest that subchondroplasty may be a promising approach for the treatment of OA with BMLs.
Bone marrow lesions (BMLs), also referred to as bone marrow edemas (BMEs), are a commonly described magnetic resonance imaging (MRI) finding associated with stress injuries, trauma, or fractures. Osteoarthritis (OA)-related BMLs represent histologically and mechanically altered subchondral bone, and have been shown to correlate with accelerated joint deterioration. ${ }^{1}$ Subchondral BMLs have been demonstrated in the knee, hip, clavicle, foot, and ankle of patients with OA. ${ }^{2-5}$ These osseous defects are unrecognized by standard radiographs, but in fat-suppressed MRI sequences they appear as diffuse water-consistent signals in the marrow space. ${ }^{1,6,7}$ BMLs occur in association with OA when physiologic subchondral remodeling fails due to ongoing joint forces, increased focalization of stress, and/or reduced healing capacity of subchondral bone. ${ }^{8,9}$ BML development is associated with localized inflammation, increased sub- chondral vascularization, high bone turnover, ${ }^{1}$ subchondral bone attrition, ${ }^{8}$ and progression of cartilage loss. ${ }^{10,11}$ Clinically, the presence of a BML closely correlates with pain (presence and severity) ${ }^{12}$ and rapid joint deterioration. ${ }^{8,10,11}$ Bone retrieval analysis of a BML reveals altered subchondral bone with loss of mechanical integrity in the region of the BML and a histologic appearance consistent with a nonhealing chronic stress fracture. ${ }^{13}$

Arthroscopic debridement generally does not provide lasting relief for patients with moderate to severe knee OA. In one prospective trial of 180 patients randomized to receive arthroscopic debridement, arthroscopic lavage, or placebo, and followed over a 24-month period, "At no point did either of the intervention groups report less pain or better function than the placebo group." 14 In another prospective trial of 172 patients randomized to receive either arthroscopic debridement/lavage received

August 3, 2015 accepted after revision

October 10, 2015

published online

December 7, 2015
DOI http://dx.doi.org/ 10.1055/s-0035-1568988. ISSN 1538-8506.
Copyright (c) 2016 by Thieme Medical Publishers, Inc., 333 Seventh Avenue, New York, NY 10001, USA. Tel: +1(212) 584-4662.
License terms




or medical/physical therapy alone, no differences in outcome were seen between the groups at 24 months in any of the six measures used to quantify pain and function. ${ }^{15}$ Finally, a recent meta-analysis of nine studies identified a small improvement in pain at 6 months after knee arthroscopy, but no improvement at 1 or 2 years. ${ }^{16}$

Natural history observations have shown that once a BML defect forms in patients with knee OA, the need for imminent total knee arthroplasty (TKA) is highly predictable. ${ }^{10,17,18}$ Additionally, BML development leads to rapid subchondral bone attrition and progressive deformity due to subchondral bone collapse. ${ }^{8}$ An observational study of patients with OA found that, compared with patients without a BML, patients with an MRI-observed BML were nearly nine times as likely to progress to TKA over a 3-year follow-up period. ${ }^{17}$

Although TKA has repeatedly been shown to be a durable intervention, leading to reliable pain relief and improved quality of life, it is a major surgical intervention and can be associated with significant recovery time and complications. ${ }^{19}$ For these reasons, less invasive, joint-preserving options are desirable, particularly for younger patients who comprise a growing proportion of patients undergoing $\mathrm{TKA}^{20,21}$ and/or those seeking to delay TKA due to long recovery times, associated costs, and/or lost productivity during the rehabilitation process. ${ }^{22-25}$

The deficiencies of existing interventions for progressive joint deterioration, and a need for therapies addressing subchondral bone pathology, have been recognized. ${ }^{26}$ Others who have investigated BMLs have theorized on the potential for an approach specifically treating compromised subchondral bone to modify symptoms. ${ }^{1}$ Because of the substantial pain and rapid bone and joint deterioration associated with a BML, an intervention with the capacity to relieve symptoms, repair subchondral bone, and alter the natural history of joint deterioration is intriguing. ${ }^{27}$ Orthobiologic treatments that mimic the strength of subchondral bone and promote bone repair represent a novel treatment concept for appropriate patients. ${ }^{28}$ Biologic solutions are also attractive due to their capacity for joint preservation; however, the orthobiologic must not alter subchondral bone properties in a way that accelerates joint deterioration or complicates arthroplasty if eventually required. ${ }^{6,9,29}$
Subchondroplasty (SCP), developed in 2007, is a procedure that utilizes an orthobiologic to treat a chronic nonhealing BML defect. ${ }^{30}$ It is performed under fluoroscopic guidance by injecting a flowable, synthetic, calcium phosphate (CaP) bone void filler ${ }^{6,9,30}$ into the region of a BML defect. SCP is often performed in conjunction with arthroscopy to improve accuracy of the desired injection location and to correct associated intra-articular pathologies (i.e., degenerative meniscus tears, loose bodies, chondral flaps, synovitis), if present. The goal of SCP is to improve the structural integrity of damaged subchondral bone and create the potential for subchondral bone remodeling. ${ }^{30,31}$ Previous reports have discussed the theoretical basis, surgical technique, and preliminary results of SCP. ${ }^{6,9,30}$ Farr and Cohen reported early findings and preliminary results for patients undergoing SCP. ${ }^{9}$ These authors noted that SCP provided a viable approach to reduce pain associated with BML, with minimal risk of significant complications. The purpose of the current study was to assess the potential of SCP combined with arthroscopy to achieve pain relief and improve function in a population of patients with BMLs associated with advanced knee OA and indications for arthroplasty.

\section{Methods}

Inclusion and exclusion criteria are listed in - Table 1.

Between May 2008 and May 2012, approximately 3,000 patients presented to the authors with indications for knee arthroplasty (i.e., moderate to severe symptoms $>2$ months and unsatisfactory response to nonoperative care) to consult with a fellowship-trained arthroplasty surgeon to discuss unicompartmental or TKA surgery. After clinical evaluation, appropriate patients were informed about SCP, including a description of the procedure and evidence limitations. Patients were considered eligible for SCP if, after MRI evaluation, they were determined to have BML in the tibia and/or femur ( - Fig. 1A,B); had pain generally localized to the same compartment as the $\operatorname{BML}(\mathrm{s})$; and met the indications for arthroplasty, including failure of weight loss, corticosteroid injections, hyaluronic acid injections, nonsteroidal antiinflammatory drugs (NSAIDs), physical therapy, and/or unloader bracing. Patients with a BML who were excluded

Table 1 Inclusion/exclusion criteria

\begin{tabular}{|c|c|}
\hline Inclusion criterion & Exclusion criterion \\
\hline $\begin{array}{l}\text { - Moderate to severe pain }>2 \text { mo } \\
\text { - Failure of symptom relief with corticosteroid injections, } \\
\text { hyaluronic acid injections, NSAIDs, physical therapy, and/or } \\
\text { unloader bracing } \\
\text { - Presence of BML(s) on MRI in a weight-bearing region of the } \\
\text { knee (medial/lateral femoral condyle or tibial plateau) } \\
\text { - Patient pain confined to the same compartment as the BML } \\
\text { - Pain in compartment of BML at least } 4 / 10 \\
\text { - Moderate to severe joint disease confined to the same } \\
\text { compartment as the BML }\end{array}$ & $\begin{array}{l}\text { - Primary cause of patient pain and loss of function due to } \\
\text { pathology other than BML, by patient history and clinical } \\
\text { evaluation } \\
\text { - Presence of gross instability } \\
\text { - }>8 \text { degrees of varus or valgus } \\
\text { - Tricompartmental radiographic grade } 4 \mathrm{OA}\end{array}$ \\
\hline
\end{tabular}

Abrreviations: BME, bone marrow edema; BML, bone marrow lesion; MRI, magnetic resonance imaging; NSAIDs, nonsteroidal anti-inflammatory drugs; OA, osteoarthritis. 

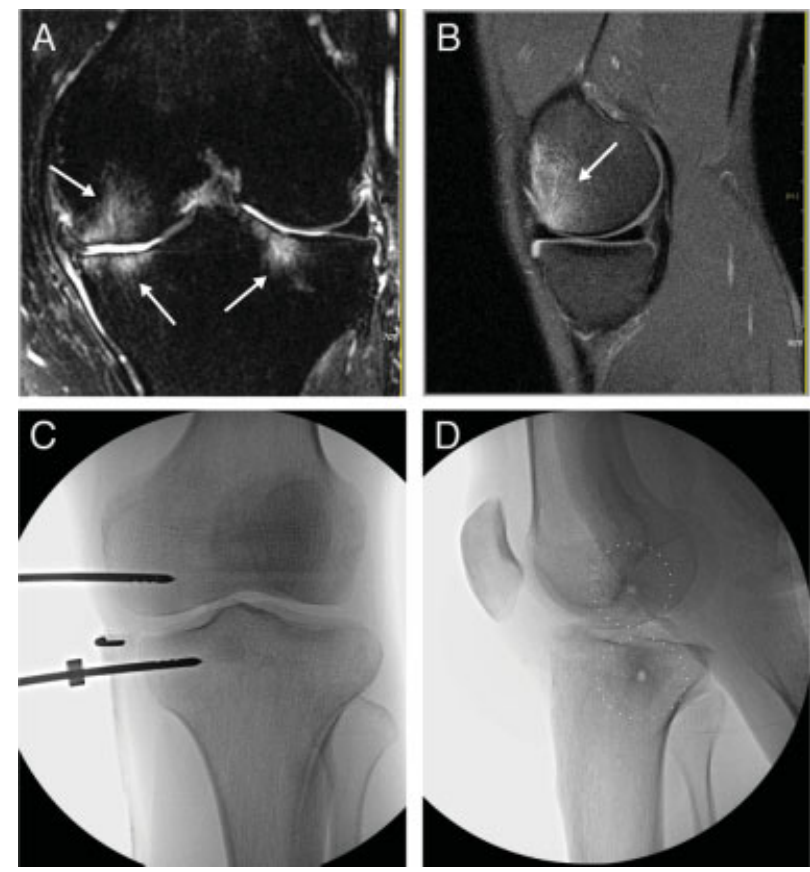

Fig. 1 Bone marrow lesions on MRI and intraoperative fluoroscopic images. (A) Coronal T2 Fat Sat MRI showing bone marrow lesions (arrows) in the medial femoral condyle and tibial plateaus. (B) Sagittal PD Fat Sat MRI showing bone marrow lesion (arrow) in the medial femoral condyle. (C) Intraoperative AP fluoroscopic image showing placement of cannulas in the medial femoral condyle and tibial plateau. (D) Intraoperative lateral fluoroscopic image after injection of CaP bone substitute (diffuse gray-shaded regions within the highlighted circles) into the medial femoral condyle and tibial plateau, with cannula bores visible in the center (white circles).

had greater than 8 degrees of varus or valgus alignment or tricompartmental radiographic Kellgren-Lawrence (K-L) grade $4 \mathrm{OA}$. Informed consent was obtained for all patients.

Patients meeting inclusion/exclusion criteria for treatment were given the option of arthroscopy combined with SCP as an alternative to arthroplasty. Of the approximately 3,000 patients entering the clinic during the study period, 69 met procedure criteria and chose SCP instead of arthroplasty.

At the outset of this retrospective study, Institutional Review Board (IRB) approval was granted by a local IRB to contact 66 of the 69 patients to review prospectivelycollected data and gather additional follow-up information. Of the three patients excluded from analysis, one patient received SCP from a surgeon not participating in the study, one received SCP treatment to the patella, and one additional patient passed away approximately 1 year following surgery of causes unrelated to their knee surgery. Thus, this cohort comprises 66 patients.

The SCP procedure was performed as described in prior publications. ${ }^{6,9,30}$ Preoperative MRI was used to determine the extent and location(s) of the BML. Intraoperative anteroposterior and lateral fluoroscopic views were used to guide the injection of the bone substitute into the desired region of the lesion ( - Fig. 1C,D). Arthroscopy was performed to aid in the accurate placement of the bone substitute, ensure that no intra-articular extravasation of the injected material occurred, evaluate intra-articular pathology, and address correctable problems (e.g., chondral flaps, loose bodies, degenerative meniscus tears, osteophytes, and synovitis). Patients with gross knee instability, or whose primary cause of pain and loss of function was due to pathology other than a BML, were excluded from the study to limit confounding factors.

Following SCP, patients were allowed to resume weightbearing activities as tolerated, with crutch assistance if needed for up to 1 week. Physical therapy was initiated 10 to 14 days after surgery, and a return to full unrestricted activities was allowed 4 to 8 weeks postoperatively. Any patient who utilized a preoperative unloader brace was given the option of using it for 4 to 8 weeks postoperatively during recovery.

Following the initial postoperative period, patients were encouraged to follow up regularly, as per standard clinical practice. When possible, patients returning for follow-up visits participated in formal clinical assessment via the visual analog scale (VAC) for pain (VAS, 10.0 representing the "worst pain ever") and/or the International Knee Documentation Committee (IKDC) Subjective Knee Evaluation Form. ${ }^{32}$ It was also determined if and when a patient later "converted" to knee arthroplasty. No VAS or IKDC scores were considered after a patient underwent arthroplasty.

For each patient, all dates were indexed to the date of the SCP. Baseline measures for both VAS and IKDC were obtained prior to SCP. Because we are primarily interested in long-term results, the "postoperative" measure used was the final measurement available in the chart for each instrument and patient. Because of variable patient participation per visit, the final IKDC measure may have been obtained at a different follow-up interval than the final VAS measure. VAS and IKDC were analyzed independently. For each, the null hypothesis was that scores were equally likely to worsen as they were to improve. We tested this null hypothesis using the binomial test (Minitab v16.1.1, Minitab Inc., State College, PA). We applied this test to the entire cohort, to patients with outcomes collected at least 6 months posttreatment, and to those with outcomes collected at least 2 years posttreatment.

\section{Results}

\section{Characteristics of the Cohort}

As summarized in - Table 2, $52 \%$ of patients were female (34 of 66), the average age was 55.9 years (range 35.0-76.0), and the average body mass index (BMI) was $30.1 \mathrm{~kg} / \mathrm{m}^{2}$ (range $20.3-53.2 \mathrm{~kg} / \mathrm{m}^{2}$ ). Prior to surgery, patients had an average of 22.4 months duration of symptoms (range 2.0-180.0 months). The modified Outerbridge grade of the chondral surface was determined during intraoperative diagnostic arthroscopy. ${ }^{33}$ Ninety-six percent (96\%) of subjects had grade 3 or 4 changes in the SCP-treated compartment, and $71 \%$ had grade 2 or less in the contralateral tibial-femoral compartment. Only two patients had less than grade 3 changes in the SCP-treated compartment (one patient with grade 0 changes, one with grade 2 changes). Sixty-two percent had grade 3 or 4 changes in the patellofemoral compartment. Patients 
Table 2 Demographic characteristics of patients with bone marrow lesion treated with subchondroplasty $(n=66)$

\begin{tabular}{|c|c|}
\hline Mean age, y (range) & $55.9(35.0-76.0)$ \\
\hline Sex (\% female) & $52 \%$ \\
\hline Mean height, in (range) ${ }^{\mathrm{a}}$ & $67.0(59.0-74.0)$ \\
\hline Mean weight, lb (range) ${ }^{a}$ & $195.0(115.0-350.0)$ \\
\hline Mean BMI, kg/m² (range) ${ }^{\mathrm{b}}$ & $30.1(20.3-53.2)$ \\
\hline Mean length of symptoms before subchondroplasty, mo (range) ${ }^{a}$ & $22.4(2.0-180.0)$ \\
\hline Side of knee, $n(\%)$ & $\begin{array}{l}\text { Left }=40(61 \%) \\
\text { Right }=26(39 \%)\end{array}$ \\
\hline Alignment, degrees varus, range ${ }^{b}$ & -8 to 8 \\
\hline $\begin{array}{l}\text { Treated area Outerbridge grade } \\
\text { Contralateral area Outerbridge grade } \\
\text { Patellofemoral Outerbridge grade }\end{array}$ & $\begin{array}{l}\text { Grade 0: } 1(2 \%) \\
\text { Grade 1: } 0(0 \%) \\
\text { Grade 2: } 1(2 \%) \\
\text { Grade 3: } 17(27 \%) \\
\text { Grade 4: } 43(69 \%) \\
\text { Grade 0: } 16(27 \%) \\
\text { Grade 1: } 4(7 \%) \\
\text { Grade 2: } 22(37 \%) \\
\text { Grade 3: } 13(22 \%) \\
\text { Grade 4: } 4(7 \%) \\
\text { Grade 0: } 7(12 \%) \\
\text { Grade 1: } 1(2 \%) \\
\text { Grade 2: } 15(25 \%) \\
\text { Grade 3: } 28(47 \%) \\
\text { Grade 4: } 9(15 \%)\end{array}$ \\
\hline Preoperative ROM, range ${ }^{a}$ & $\begin{array}{l}\text { Extension: } 0-5 \text { degrees } \\
\text { Flexion: } 100-135 \text { degrees }\end{array}$ \\
\hline $\begin{array}{l}\text { Prior treatments, } n \text { (\%) } \\
\text { Arthroscopy } \\
\text { Bracing } \\
\text { Cortisone } \\
\text { Hyaluronic acid } \\
\text { NSAID } \\
\text { Partial medial meniscectomy } \\
\text { Physical therapy }\end{array}$ & $\begin{array}{l}19(29 \%) \\
14(21 \%) \\
41(62 \%) \\
48(73 \%) \\
30(45 \%) \\
18(27 \%) \\
8(12 \%)\end{array}$ \\
\hline Baseline VAS scores, mean (SD, range) ${ }^{c}$ & $7.6(1.5,4-10)$ \\
\hline Baseline IKDC scores, mean (SD, range) ${ }^{c}$ & $30.5(10,14.9-55.2)$ \\
\hline
\end{tabular}

Abbreviations: BMI, body mass index; IKDC, International Knee Documentation Committee; NSAID, nonsteroidal anti-inflammatory drug; OA, osteoarthritis; ROM, range of motion; SD, standard deviation; VAS, visual analog scale.

a $<5 \%$ missing data points.

${ }^{\mathrm{b}}<10 \%$ missing data points.

${ }^{\mathrm{c}}<15 \%$ missing data points.

described mean preoperative pain as a 7.6 out of 10 (range 410). Mean IKDC score at baseline was 30.5 (range 14.9-55.2).

\section{Durable Improvement in Pain Scores after Subchondroplasty with Arthroscopy}

Preoperative (baseline) VAS scores were available for 59 out of the 66 patients (89\%), and at least one postoperative VAS score was available for 57 of these 59 (median postoperative time of final follow-up VAS = 27.2 months). As illustrated in - Fig. 2A, 50 of these 57 patients exhibited pain improvement on final follow-up, 3 had worse pain scores, and 4 were unchanged. Notably, even those patients who ultimately elected to receive arthroplasty (15 out of these 57 patients) typically showed improved pain scores (see Discussion). - Fig. 2B illustrates that improvements in pain scores were observed at all durations post-SCP.

The binomial test rejected the null hypothesis that pain was not improved after SCP, both across all patients $(<0.001)$, or considering only those whose last follow-up VAS was at least 6 months postoperative $(n=44,38$ improved, $p<0.001$ ), or at least 2 years postoperative follow-up ( $n=34,29$ improved, $p<0.001$ ). Thus, patients experienced durable pain relief after SCP.

The magnitude of improvement in VAS pain scores was clinically meaningful. Across the three groupings (all patients, those with at least 6-month follow-up VAS and those with at least 2-year follow-up VAS), the mean improvements in VAS scores were 4.2, 4.3, and 4.5 points, respectively. For VAS pain 

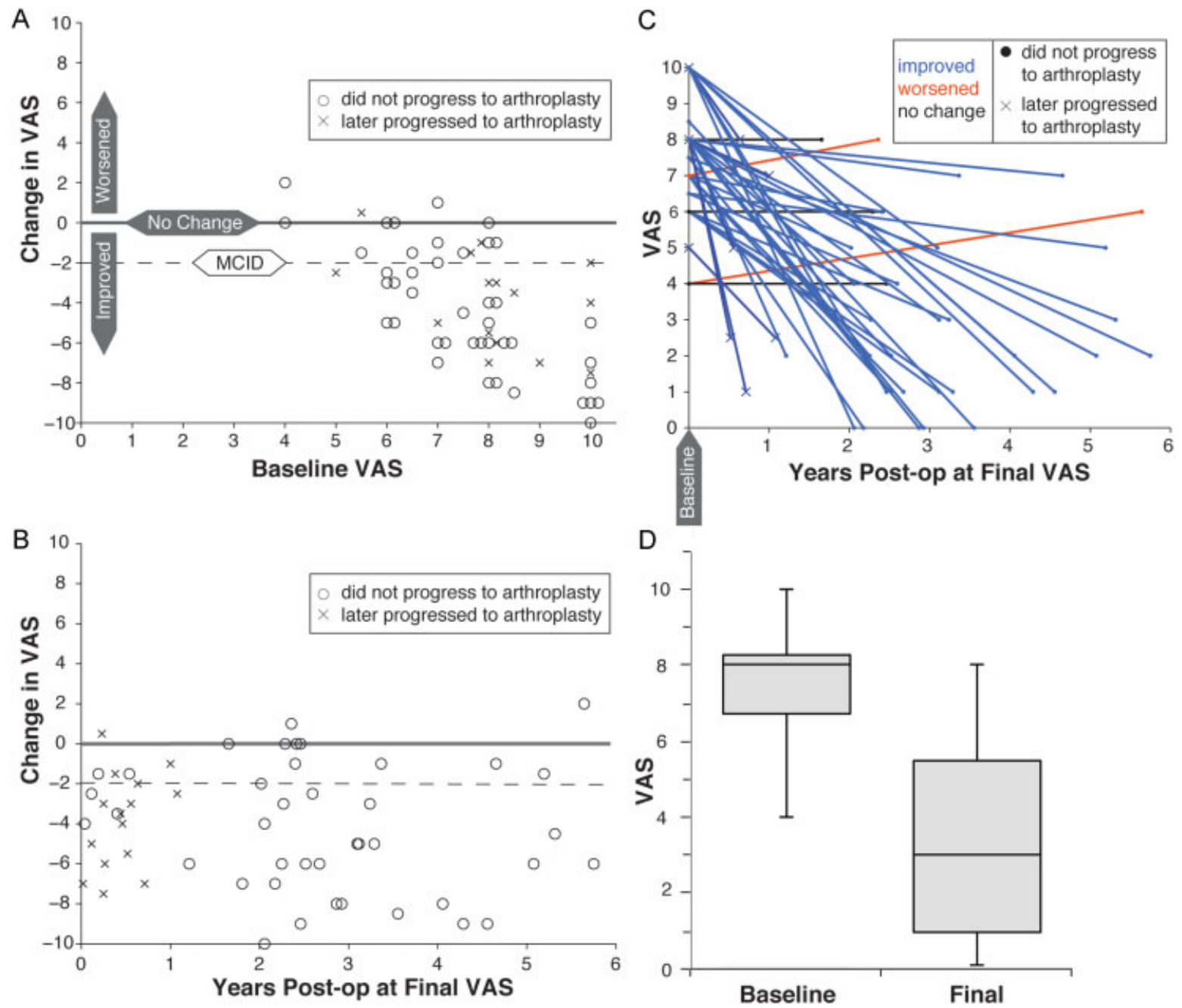

Fig. 2 Improvement of visual analog scale (VAS) pain scores after subchondroplasty. (A) Change in VAS scores (final minus baseline) for all 57 patients who had both a presurgical score and at least one follow-up score. (B) Change in VAS score, plotted versus the number of years after subchondroplasty at which the final follow-up VAS score was obtained, for each patient. (C) Baseline and final VAS scores, for all patients whose final follow-up VAS score was at least 6 months postsurgery $(n=44)$. (D) Box-and-whiskers plots of baseline and final VAS scores, showing median, interquartiles, and range (whiskers). MCID, minimal clinically important differences.

scores, an improvement of 2 points (20 $\mathrm{mm}$ on the $100-\mathrm{mm}$ scale) is considered clinically important (minimal clinically important differences [MCID]). ${ }^{34}$

-Fig. $2 \mathrm{C}$ shows all three dimensions of the data (baseline VAS, final VAS, and postoperative duration at final follow-up) for all patients with at least 6 months of VAS follow-up. - Fig. 2D shows the distributions of scores in this patient group.

\section{Durable Improvement in IKDC (symptom/function) Scores after Subchondroplasty with Arthroscopy}

Preoperative (baseline) IKDC scores were available for 48 patients, all of whom also had at least one postoperative score. As illustrated in -Fig. 3A, 38 of these 48 patients exhibited improvement (higher IKDC scores indicate improved symptoms and function) on final follow-up, 9 had worse scores, and 1 was unchanged. -Fig. 2B illustrates that improvements in IKDC scores were observed at all durations post-SCP.
The binomial test rejected the null hypothesis that function was not improved after SCP, both across all patients $(p<0.001)$, or considering only those whose last follow-up IKDC was at least 6 months postoperative $(n=35,28$ improved, $p<0.001$ ), or at least 2 years postoperative ( $n=26,21$ improved, $p<0.002$ ). Thus, patients experienced durable functional/symptomatic improvement after SCP.

The magnitude of improvement in IKDC scores was clinically meaningful. Across the three groupings (all patients, those with at least 6 months follow-up IKDC, those with at least 2-year follow-up IKDC), the mean improvements in IKDC scores were $18.3,17.2$, and 17.8 points, respectively. For IKDC pain scores, an improvement of 11.5 points is considered clinically important (MCID). ${ }^{35}$

-Fig. $3 C$ shows all three dimensions of the data (baseline IKDC, final IKDC, and postoperative duration at final follow-up) for all patients with at least 6 months of IKDC follow-up. - Fig. 3D shows the distributions of IKDC scores in this patient group. 

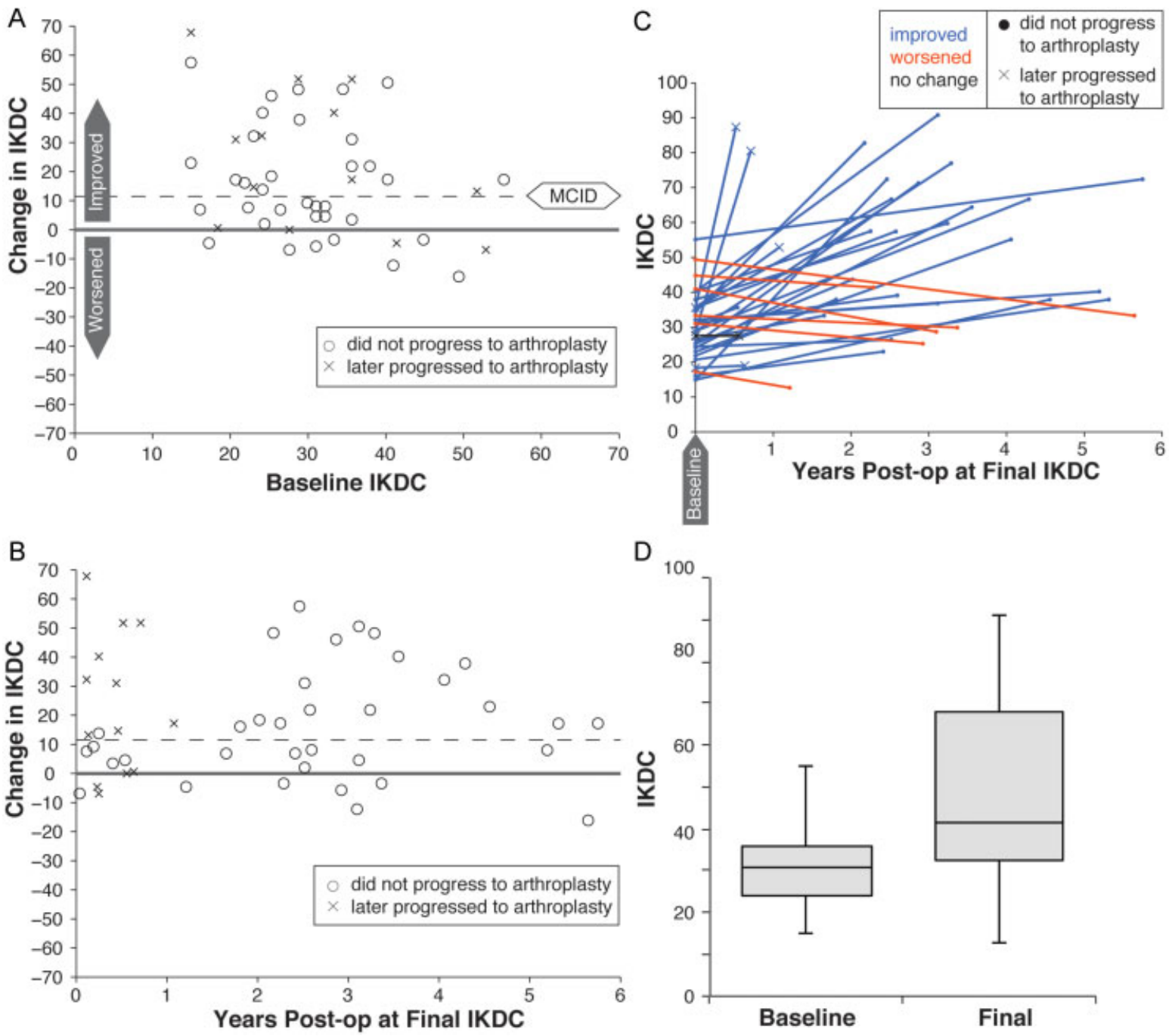

Fig. 3 Improvement of International Knee Documentation Committee (IKDC) function/pain scores after subchondroplasty. (A) Change in IKDC scores (final minus baseline) for all 57 patients who had both a presurgical score and at least one follow-up score. (B) Change in IKDC score, plotted versus the number of years after subchondroplasty at which the final follow-up IKDC score was obtained, for each patient. (C) Baseline and final IKDC scores, for all patients whose final follow-up IKDC score was at least 6 months postsurgery $(n=44)$. (D) Box-and-whiskers plots of baseline and final IKDC scores, showing median, interquartiles, and range (whiskers).

\section{Conversion to Arthroplasty}

Sixty of the 66 patients (91\%) were available for 2-year followup that allowed a determination of whether and when patients elected to undergo arthroplasty. Of the six patients not available, only one had 1-year follow-up data, and five were lost to follow-up. Kaplan-Meier analysis (-Fig. 4), demonstrated 2-year joint preservation survivorship of $70 \%$ (42 out of 60 ) for study patients. Because patients in this study initially presented for arthroplasty consultation, this survival rate seems promising.

Variables potentially associated with conversion to arthroplasty were assessed using logistic regression and included: patient age, BMI, length of symptoms, joint alignment, preoperative VAS scores, treated area grade, previous partial meniscectomy, and the presence of kissing lesions (adjacent BMLs of the tibia and femur). Older age and a history of prior meniscectomy were both positively associated with subsequent conversion to arthroplasty, with or without controlling

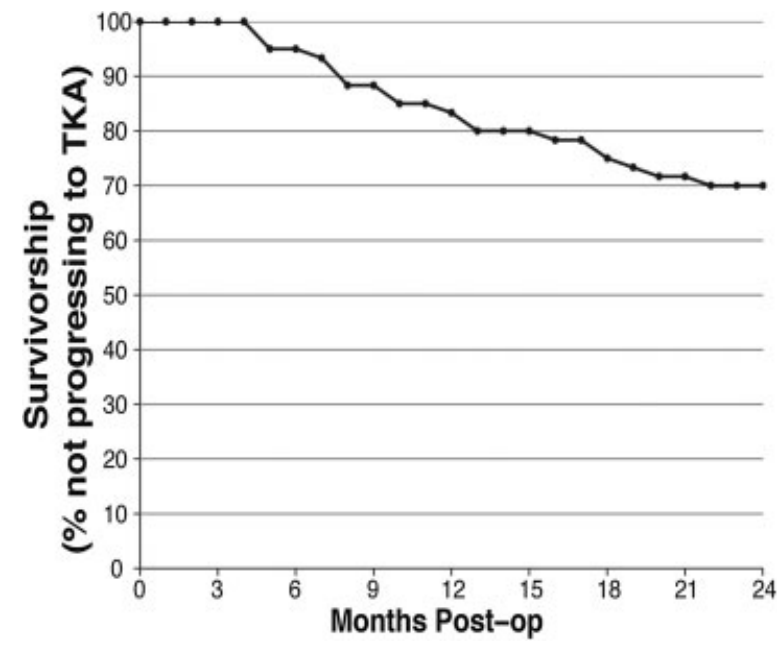

Fig. 4 Kaplan-Meier plot of conversion to total knee arthroplasty (TKA). At 2 years after subchondroplasty, $70 \%$ of patients (42/60) did not still elect to receive arthroplasty on the affected knee. 
for length of symptoms and BMI $(p<0.05)$. The mean age of patients who converted to arthroplasty was 58.2 years (range 47.0-76.0), compared with a mean of 55.1 years (range 35.073.0) among patients who did not. Thirty-nine percent (39\%) of patients who had subsequent arthroplasty also had a previous partial meniscectomy, compared with $23 \%$ of patients who did not. Other variables analyzed showed no significant association with the probability of conversion.

Adverse Events. The observed number of adverse events following the SCP procedure included one patient who experienced postoperative drainage at the CaP injection site, which resolved with surgical irrigation and debridement, and one patient diagnosed postoperatively with a deep vein thrombosis, which required treatment with oral anticoagulation.

\section{Discussion}

The natural history of $\mathrm{OA}$ is slow but progressive joint degeneration. Knee OA patients with BMLs have a poor prognosis, with accelerated progression to the need for total knee replacement. $8,10,11$ Considering these facts, the magnitude and durability of the pain relief and functional improvement observed in this retrospective study is noteworthy. For patients with at least 2 years of follow-up, mean pain improvement was 4.5 points on the VAS scale (corresponding to "good to excellent" pain relief $^{36}$ ), and mean functional/ symptomatic improvement was 17.8 points on the IKDC scale (relative to the MCID of 11.5). Our results suggest that SCP may be a promising treatment for BMLs associated with OA.

Several limitations of this study warrant discussion. First, with any nonrandomized study, selection bias is possible. For example, qualifying patients were allowed to select between arthroplasty and the alternative treatment under study (SCP). It is possible that an unidentified personal trait could predis- pose someone to both (1) choose SCP over arthroplasty and (2) exhibit sustained improvement in knee pain for reasons unrelated to SCP. Mitigating this concern is the poor prognosis of patients entering this study, who were all originally indicated for arthroplasty. A second limitation is a lack of standardization in the collection of follow-up data. Not all subjects completed the patient reported outcomes measures at all time points, and the final follow-up data were collected at varying time points. This makes it difficult to evaluate the progression of pain and function outcomes for patients treated with SCP from this dataset. A third limitation is a lack of postoperative imaging to assess changes in the BML. Future studies that include a series of MR images postoperatively may provide valuable insight into the remodeling of the $\mathrm{CaP}$ material and potential relationships between the status of the BML and patient outcome. A fourth limitation is the absence of control cohorts: there was no placebo cohort, and because SCP was always performed along with arthroscopy, the relative contributions of these two procedures were not empirically separated. Future study with an arthroscopymatched control group may be useful in further evaluating efficacy. However, several studies of subjects with pathology and symptoms similar to the present patient population indicate that arthroscopic debridement alone provides no durable relief of OA symptoms ( - Table 3 ). The lack of durable pain relief in these studies may have been due to the presence of BMLs. BMLs are commonly present in this patient population and are not treated by traditional arthroscopy.

Another recent study evaluated the effect of SCP on outcomes in patients with knee BMLs, ${ }^{37}$ in a nonmatched cohort of 22 patients with knee OA. They reported that both the Knee Injury and Osteoarthritis Outcome Score (KOOS) and the Tegner-Lysholm Knee Scoring Scale scores significantly improved $(p<0.001)$ at greater than 6 months posttreatment. Surprisingly, despite these improvements, the authors

Table 3 Review of studies quantifying the effects of knee arthroscopy on pain and function outcomes at 24 months

\begin{tabular}{|c|c|c|c|}
\hline Study & Patients & Design & Outcome \\
\hline $\begin{array}{l}\text { Moseley et al, New Engl J Med, } \\
2002\end{array}$ & $\begin{array}{l}\text { - } n=180 \text { patients } \\
\text { - VAS pain } \geq 4 \\
\text { - No severe joint deformity }\end{array}$ & $\begin{array}{l}\text { - Prospective, randomized to } \\
\text { 1. Arthroscopic debride- } \\
\text { ment } \\
\text { 2. Arthroscopic lavage } \\
\text { 3. Placebo } \\
\text { - Follow-up through } 24 \text { mo }\end{array}$ & $\begin{array}{l}\text { No differences in pain or } \\
\text { function ( } 6 \text { different score } \\
\text { metrics) in any groups at any } \\
\text { time point }\end{array}$ \\
\hline $\begin{array}{l}\text { Kirkley et al, New Engl J Med, } \\
2008\end{array}$ & $\begin{array}{l}\text { - } n=172 \text { patients } \\
\text { - Kellgren-Lawrence grade } 2 \text {, } \\
\quad 3, \text { or } 4\end{array}$ & $\begin{array}{l}\text { - Prospective, randomized to } \\
\text { 1. Arthroscopic debride- } \\
\text { ment and lavage to- } \\
\text { gether with optimized } \\
\text { physical and medical } \\
\text { therapy } \\
\text { 2. Physical and medical } \\
\text { therapy alone } \\
\text { - Follow-up through } 24 \text { mo }\end{array}$ & $\begin{array}{l}\text { No differences in WOMAC or } \\
\text { SF-36 scores between groups }\end{array}$ \\
\hline Thorlund et al, BMJ, 2015 & $\begin{array}{l}\text { - } n=1,270 \text { patients across } 9 \\
\text { studies }\end{array}$ & $\begin{array}{l}\text { - Meta-analysis } \\
\text { - Follow up through } 24 \text { mo }\end{array}$ & $\begin{array}{l}\text { Small improvements at } 3 \text { and } \\
6 \text { mo in pain } \\
\text { No improvement in pain or } \\
\text { function at } 12 \text { and } 24 \text { mo }\end{array}$ \\
\hline
\end{tabular}


concluded that the treatment was "ineffective." However, the authors defined "clinical failure" based on a categorization of postoperative Tegner-Lysholm scores historically used to evaluate success of anterior cruciate ligament (ACL) reconstruction, a scoring method likely inappropriate for evaluating the success rate of this treatment. ${ }^{38,39}$

The present study is the largest series to date evaluating the effectiveness of SCP. Interestingly, although most patients (70\%) did not convert to arthroplasty, a majority of the patients who did progress to arthroplasty actually had shown improvements in both VAS and IKDC scores prior to their decision to undergo a total knee replacement. Factors such as patient expectations (both with SCP and total knee replacement) and patient satisfaction were not evaluated in this study. This highlights the complexity of the personal decision to undergo surgery and thus the difficulty of using revision to an elective surgery for assessing efficacy.

It is important to consider biologic options for subchondral bone lesion treatment, such as calcium-phosphate SCP, in the context of other treatment options. TKA provides predictable pain relief; however, functionality is typically reduced. $^{22,23,25,30}$ Additionally, TKA is costly, invasive, and requires substantial recovery time (typically, 6-12 months). ${ }^{24,40}$ SCP, on the other hand, represents a minimally invasive approach that provides fairly reliable pain relief while preserving the native joint, and enables patients to resume some normal activities as soon as 1 week postprocedure. $^{30}$ These factors may be particularly important for younger, active patients who wish to reduce pain and avoid arthroplasty, but retain function and delay the productivity losses associated with major surgery.

\section{Conclusion}

Patients with severe knee OA have limited options, usually requiring arthroplasty to regain mobility and relieve pain. For patients who also have BMLs, the prognosis is poor and joint deterioration usually progresses rapidly. In this study we evaluated the efficacy of using SCP-a technique for applying a CaP bone substitute to the BML defect-with arthroscopy, as a less invasive, joint-preserving option in patients with BMLs associated with advanced OA. We observed clinically significant and durable improvements in pain and function in most patients in our investigation. Understanding the limitations of retrospective case studies, this first patient series shows potential for treating patients with pain due to presence of BMLs.

\section{References}

1 Eriksen EF, Ringe JD. Bone marrow lesions: a universal bone response to injury? Rheumatol Int 2012;32(3):575-584

2 Radke S, Kirschner S, Seipel V, Rader C, Eulert J. Treatment of transient bone marrow oedema of the hip-a comparative study. Int Orthop 2003;27(3):149-152

3 Hofmann S, Engel A, Neuhold A, Leder K, Kramer J, Plenk H Jr. Bonemarrow oedema syndrome and transient osteoporosis of the hip. an MRI-controlled study of treatment by core decompression. J Bone Joint Surg Br 1993;75(2):210-216
4 Shubin Stein BE, Ahmad CS, Pfaff $\mathrm{CH}$, Bigliani LU, Levine WN. A comparison of magnetic resonance imaging findings of the acromioclavicular joint in symptomatic versus asymptomatic patients. J Shoulder Elbow Surg 2006;15(1):56-59

5 Orr JD, Sabesan V, Major N, Nunley J. Painful bone marrow edema syndrome of the foot and ankle. Foot Ankle Int 2010;31(11):949-953

6 Cohen SB, Sharkey PF. Surgical treatment of osteoarthritis pain related to subchondral bone defects or bone marrow lesions: subchondroplasty. Tech Knee Surg 2012;11(4):170-175

7 Jimenez-Boj E, Nöbauer-Huhmann I, Hanslik-Schnabel B, et al. Bone erosions and bone marrow edema as defined by magnetic resonance imaging reflect true bone marrow inflammation in rheumatoid arthritis. Arthritis Rheum 2007;56(4):1118-1124

8 Roemer FW, Neogi T, Nevitt MC, et al. Subchondral bone marrow lesions are highly associated with, and predict subchondral bone attrition longitudinally: the MOST study. Osteoarthritis Cartilage 2010;18(1):47-53

9 Farr J II, Cohen SB. Expanding applications of the subchondroplasty procedure for the treatment of bone marrow lesions observed on magnetic resonance imaging. Oper Tech Sports Med 2013;21(2):138-143

10 Tanamas SK, Wluka AE, Pelletier JP, et al. Bone marrow lesions in people with knee osteoarthritis predict progression of disease and joint replacement: a longitudinal study. Rheumatology (Oxford) 2010;49(12):2413-2419

11 Wluka AE, Wang Y, Davies-Tuck M, English DR, Giles GG, Cicuttini FM. Bone marrow lesions predict progression of cartilage defects and loss of cartilage volume in healthy middle-aged adults without knee pain over 2 yrs. Rheumatology (Oxford) 2008;47(9): 1392-1396

12 Felson DT, Chaisson CE, Hill CL, et al. The association of bone marrow lesions with pain in knee osteoarthritis. Ann Intern Med 2001;134(7):541-549

13 Taljanovic MS, Graham AR, Benjamin JB, et al. Bone marrow edema pattern in advanced hip osteoarthritis: quantitative assessment with magnetic resonance imaging and correlation with clinical examination, radiographic findings, and histopathology. Skeletal Radiol 2008;37(5):423-431

14 Moseley JB, O'Malley K, Petersen NJ, et al. A controlled trial of arthroscopic surgery for osteoarthritis of the knee. N Engl J Med 2002;347(2):81-88

15 Kirkley A, Birmingham TB, Litchfield RB, et al. A randomized trial of arthroscopic surgery for osteoarthritis of the knee. N Engl J Med 2008;359(11):1097-1107

16 Thorlund JB, Juhl CB, Roos EM, Lohmander LS. Arthroscopic surgery for degenerative knee: systematic review and metaanalysis of benefits and harms. BMJ 2015;350:h2747

17 Scher C, Craig J, Nelson F. Bone marrow edema in the knee in osteoarthrosis and association with total knee arthroplasty within a three-year follow-up. Skeletal Radiol 2008;37(7):609-617

18 Kröner AH, Berger CE, Kluger R, Oberhauser G, Bock P, Engel A. Influence of high tibial osteotomy on bone marrow edema in the knee. Clin Orthop Relat Res 2007;454(454):155-162

19 Healy WL, Della Valle CJ, Iorio R, et al. Complications of total knee arthroplasty: standardized list and definitions of the Knee Society. Clin Orthop Relat Res 2013;471(1):215-220

20 Kurtz S, Mowat F, Ong K, Chan N, Lau E, Halpern M. Prevalence of primary and revision total hip and knee arthroplasty in the United States from 1990 through 2002. J Bone Joint Surg Am 2005;87(7): 1487-1497

21 Kurtz SM, Lau E, Ong K, Zhao K, Kelly M, Bozic KJ. Future young patient demand for primary and revision joint replacement: national projections from 2010 to 2030. Clin Orthop Relat Res 2009;467(10):2606-2612

22 Franklin PD, Li W, Ayers DC. The Chitranjan Ranawat Award: functional outcome after total knee replacement varies with patient attributes. Clin Orthop Relat Res 2008;466(11):2597-2604 
23 Mizner RL, Petterson SC, Clements KE, Zeni JA Jr, Irrgang JJ, SnyderMackler L. Measuring functional improvement after total knee arthroplasty requires both performance-based and patient-report assessments: a longitudinal analysis of outcomes. J Arthroplasty 2011;26(5):728-737

24 Browne JP, Bastaki H, Dawson J. What is the optimal time point to assess patient-reported recovery after hip and knee replacement? A systematic review and analysis of routinely reported outcome data from the English patient-reported outcome measures programme. Health Qual Life Outcomes 2013;11:128

25 Singh JA, O'Byrne M, Harmsen S, Lewallen D. Predictors of moderate-severe functional limitation after primary total knee arthroplasty (TKA): 4701 TKAs at 2 years and 2935 TKAs at 5 years. Osteoarthritis Cartilage 2010;18(4):515-521

26 Mastbergen SC, Lafeber FP. Changes in subchondral bone early in the development of osteoarthritis. Arthritis Rheum 2011;63(9): 2561-2563

27 Lim YZ, Wang Y, Wluka AE, et al. Association of obesity and systemic factors with bone marrow lesions at the knee: a systematic review. Semin Arthritis Rheum 2014;43(5):600-612

28 Moore WR, Graves SE, Bain GI. Synthetic bone graft substitutes. ANZ J Surg 2001;71(6):354-361

29 Tofighi A, Rosenberg A, Sutaria M, Balata S, Chang J. New generation of synthetic, bioresorbable and injectable calcium phosphate bone substitute materials: Alpha-bsm ${ }^{\circledR}$, BetabsmTM and Gamma-bsmTM. J Biomimet Biomat Tissue Engineer 2009;2:39-55

30 Sharkey PF, Cohen SB, Leinberry CF, Parvizi J. Subchondral bone marrow lesions associated with knee osteoarthritis. Am J Orthop 2012;41(9):413-417

31 Bajammal SS, Zlowodzki M, Lelwica A, et al. The use of calcium phosphate bone cement in fracture treatment. A meta-analysis of randomized trials. J Bone Joint Surg Am 2008;90(6):1186-1196
32 Higgins LD, Taylor MK, Park D, et al; International Knee Documentation Committee. Reliability and validity of the International Knee Documentation Committee (IKDC) Subjective Knee Form. Joint Bone Spine 2007;74(6):594-599

33 Browne JE, Branch TP. Surgical alternatives for treatment of articular cartilage lesions. J Am Acad Orthop Surg 2000;8(3):180-189

34 Tubach F, Ravaud P, Baron G, et al. Evaluation of clinically relevant changes in patient reported outcomes in knee and hip osteoarthritis: the minimal clinically important improvement. Ann Rheum Dis 2005;64(1):29-33

35 Irrgang JJ, Anderson AF, Boland AL, et al; International Knee Documentation Committee. Responsiveness of the International Knee Documentation Committee Subjective Knee Form. Am J Sports Med 2006;34(10):1567-1573

36 Grilo RM, Treves R, Preux PM, Vergne-Salle P, Bertin P. Clinically relevant VAS pain score change in patients with acute rheumatic conditions. Joint Bone Spine 2007;74(4):358-361

37 Chatterjee D, McGee A, Strauss E, Youm T, Jazrawi L. Subchondral calcium phosphate is ineffective for bone marrow edema lesions in adults with advanced osteoarthritis. Clin Orthop Relat Res 2015; 473(7):2334-2342

38 Mitsou A, Vallianatos P, Piskopakis N, Maheras S. Anterior cruciate ligament reconstruction by over-the-top repair combined with popliteus tendon plasty. J Bone Joint Surg Br 1990; 72(3):398-404

39 Bengtsson J, Möllborg J, Werner S. A study for testing the sensitivity and reliability of the Lysholm knee scoring scale. Knee Surg Sports Traumatol Arthrosc 1996;4(1):27-31

40 U.S. Agency for Healthcare Research and Quality. National and regional estimates on hospital use for all patients from the HCUP Nationwide Inpatient Sample (NIS) for arthroplasty of knee (surgical reconstruction or replacement of knee). 2012. http:// hcupnet.ahrq.gov/HCUPnet.jsp 